\title{
A LOGICAL FRAMEWORK FOR COALITIONAL EFFECTIVITY IN DYNAMIC PROCEDURES
}

\author{
Marc Pauly \\ CWI, The Netherlands
}

\begin{abstract}
Effectivity frames are introduced as a model of what groups of agents can achieve by coordinated action in dynamic processes such as extensive games with or without simultaneous moves. Local effectivity is distinguished from different kinds of global and terminal effectivity, respectively what groups of players can maintain throughout and what they can achieve eventually. Examples are provided of how effectivity frames can be used as a tool (1) to model complex multi-agent processes such as voting procedures, (2) to investigate the interplay between local and global properties of these dynamic procedures, and (3) to examine whether a particular effectivity function can be implemented or realized by a dynamic procedure of a specific type. Finally, a modal logic for local and global coalitional effectivity is presented, and it is shown how, for example, a realization question can be translated into this logical framework yielding a satisfiability problem.
\end{abstract}

\section{INTRODUCTION}

Modelling actions and their effects is a task which has occupied many researchers in computer science, logic, economics and philosophy. In the simplest case, we have one agent (person, process) who can choose between taking different actions which change the state of the world in various ways. A simple model of this scenario will contain an accessibility relation $R$ which associates to every state of the world all those states which the agent can bring about through his actions; i.e. sRt 
holds if the agent can act in state $s$ as to bring about state $t$. In modal logic, one introduces a language to talk about such Kripke models: $\diamond \varphi$ expresses that the agent can act in such a way that $\varphi$ will be true after his action.

When generalized to multiple agents, Kripke models do not suffice anymore. Instead I will employ effectivity functions (Moulin and Peleg, 1982; Abdou and Keiding, 1991) which associate to every state and every group of agents $C$ the sets of states for which $C$ is effective. Since the resulting states can again be associated with an effectivity function, I obtain a dynamic model of what groups of agents can achieve by joint action. At each state of the model, local effectivity can be distinguished from different kinds of terminal effectivity: While a group of agents may not be able to bring about $\varphi$ immediately, the group may be able to achieve $\varphi$ eventually. As I show in Section III, extensive games, nondeterministic processes and voting procedures can all be viewed as instances of these general effectivity frames. Section IV illustrates some applications of the model; e.g. to a simple voting scenario. To describe effectivity frames, I use a modal logic presented in Section V. Properties of effectivity frames can be expressed as modal axioms, and questions about the existence of a particular procedure implementing a given effectivity function can be expressed as satisfiability problems. While I do not study the logic itself in the present paper, comments on some of its meta-theoretic properties are made in Section VI.

A fragment of the logic discussed in this paper has first been introduced in Pauly (2000) which only dealt with local effectivity. The present paper deals also with global and terminal effectivity, witnessed by the addition of the $\left[C^{*}\right] \varphi$ modality to the logic. As will be argued, especially terminal effectivity is a concept which can be fruitfully applied in modelling, e.g. multistage voting procedures.

\section{A DYNAMIC MODEL OF EFFECTIVITY}

\section{II.1. Effectivity functions}

Throughout this paper, I assume that a nonempty finite set $N$ of agents or players is given, as well as a nonempty set of states $S$. An effectivity finction $E: \mathscr{P}(N) \rightarrow \mathscr{P}(\mathscr{S}(S))$ associates to every group of players the sets of outcomes for which the group is effective. For a coalition $C \subseteq N$, $X \in E(C)$ will hold iff the players in $C$ have a joint strategy for bringing about an outcome in $X$. We shall require that effectivity functions are monotonic; i.e. $X \subseteq Y \subseteq S$ implies that for every coalition $C \subseteq N$, if $X \in E(C)$ then $Y \in E(C)$. Given the intuitions put forth, this requirement should be a natural one.

(C) Blackwell Publishers Lid and the Board of Truslecs of the Bulletin of Economic Rescarch 2001. 
I present some further properties of effectivity functions which will play a role later: In many circunstances one will want to assume that a group which becomes larger has possibly more power but certainly not less. In that case, $E$ is coalition-monotonic i.e. for $C \subseteq C^{\prime} \subseteq N$, $E(C) \subseteq E\left(C^{\prime}\right)$. As a basic consistency requirement, we usually want to exclude cases where complementary coalitions are effective for complementary things, for in that case, both coalitions could use their power and end up in an inconsistent situation. The notion of regularity captures this concern: $E$ is $C$-regular if for all $X$, if $X \in E(C)$ then $\bar{X} \notin E(\bar{C})$. As a converse to regularity, call $E$ C-maximal if for all $X$, if $\bar{X} \notin E(\bar{C})$ then $X \in E(C)$. $E$ is regular (maximal) iff for all coalitions $C$ it is $C$-regular $(C$ maximal). If we think of a two-player game with two possible outcomes win ${ }_{1}$ and winn, regularity expresses that the game is zero-sum: If player 1 wins $\left(\{1\}\right.$ is effective for $\left.\left\{w_{i n}\right\}\right)$ then player 2 cannot win $(\{2\}$ is not effective for $\left\{\right.$ win $\left._{2}\right\}$ ). Sinilarly, maximality expresses determinacy: If player 1 does not win $\left(\{1\}\right.$ is not effective for $\left\{\right.$ win $\left.\left._{1}\right\}\right)$ then player 2 must win $\left(\{2\}\right.$ is effective for $\left.\left\{w_{i} n_{2}\right\}\right)$. Finally, the most interesting principle governs the formation of coalitions. It states that coalitions can combine their stratcgies to (possibly) achieve more: $E$ is superadditive if for all $X_{1}, X_{2}, C_{1}, C_{2}$ such that $C_{1} \cap C_{2}=\varnothing, X_{1} \in E\left(C_{1}\right)$ and $X_{2} \in E\left(C_{2}\right)$ imply that $X_{1} \cap X_{2} \in E\left(C_{1} \cup C_{2}\right)$.

Effectivity functions have been investigated by, for example, Moulin and Peleg (1982), Abdou and Keiding (1991) and Peleg (1998), and they find application in the theory of social choice (Moulin, 1983) where the agents are voters who try to force certain election outcomes. While effectivity functions are too crude to capture all aspects of a situation in which different agents interact (sce also Section IV.3), they do capture the essential information about what power different groups of agents have. In the voting example to be discussed in Section IV.2, effectivity functions capture the allocation of veto power to individuals and coalitions. This analysis thus centres on what individuals and groups can achieve, independent of what they prefer to achieve. Clearly, in order to predict what agents will do in a particular strategic situation, their preferences need to be taken into account, and this could be done using the notion of the core of effectivity functions. I will not pursue this line of investigation here since I feel that even without incorporating players' preferences, the study of coalitional effectivity can yield some interesting results.

While we consider any monotonic function $E: \mathscr{P}(N) \rightarrow \mathscr{P}(\mathscr{P}(S))$ to be an effectivity function, most of the literature has taken a more restrictive view, requiring $E$ to satisfy various basic properties. The choice of these basic properties, however, is somewhal arbitrary, and this opinion is supported by the fact that authors differ in which basic properties they require. Here, I decided to be as general as possible regarding the notion of an effectivity function, requiring only monotonicity which is needed to

ii) Blackwell Publishers Lud and the Board of Truslecs of the Bulletin of Economic Rescarch 2001. 
obtain proper definitions for the global and terminal effectivity functions to be defined below.

\section{II.2. Effectivity frames and structures}

The dynamic models of effectivity are captured by the notion of an effectivity frame $g_{r}=(S, E)$, where $S$ is the nonempty set of states and $E: S \rightarrow(\mathscr{P}(N) \rightarrow \mathscr{P}(\mathscr{P}(S)))$ is the local effectivity structure which associates to every state $s \in S$ an effectivity function $E(s)$; i.e. $X \in E(s)(C)$ if at state $s$, coalition $C$ is effective for the set of states $X$. Given that effectivity functions are linked to states and that the alternatives which can be brought about are states again, frames do present a dynamic model of effectivity. For easier readability, we shall often write $s E_{C} X$ instead of $X \in E(s)(C)$, and we will sometimes view the effectivity structure $E$ as a collection of maps $E_{C}: \Phi(S) \rightarrow \Phi(S)$ with $E_{C}(X)=$ $\left\{s \in S \mid s E_{C} X\right\}$. Monotonicity can now be formulated as the requirement that if $X \subseteq X^{\prime}$ then $E_{C}(X) \subseteq E_{C}\left(X^{\prime}\right)$. A state $s$ where no coalition is effective for anything is called a terminal state; i.e. $s$ is terminal iff there is no coalition $C$ and no set of states $X$ such that $s E_{C} X$. We denote the set of terminal states $\left\{s \in S \mid \neg \exists C \exists Y s E_{C} Y\right\}$ as $S_{\perp}$.

Lastly, note that all of the properties of effectivity functions introduced previously can be lifted to frames/structures: a frame $(S, E)$ has a given property (e.g. maximality) iff every $E(s)$ has it.

\section{II.3. Global and terminal effectivity}

Given an effectivity frame $\mathscr{F}=(S, E)$ which contains information about effectivity at every state, we can also investigate effectiveness in the long run. For this, we define the global effectivity structure $E^{*}: S \rightarrow$ $(\mathscr{P}(N) \rightarrow \mathscr{S}(\mathscr{P}(S)))$, where a coalition $C$ is globally effective for $X$ if it can maintain $X$ throughout the game/process. Again, the global effectivity structure determines a (global) effectivity function $E^{*}(s)$ for every state $s \in S$. Viewing $E_{C}^{*}$ as a function from $\mathscr{P}(S)$ to $\mathscr{P}(S)$, we define:

$$
E_{C}^{*}(Z)=\bigcup\left\{X \subseteq S \mid X \subseteq Z \cap\left(S_{\perp} \cup E_{C}(X)\right)\right\} .
$$

By the Knaster-Tarski theorem, since $E_{C}$ is a monotonic operator, $E_{C}^{*}(Z)$ is the greatest fixpoint of the operator $F_{C}^{Z}(X)=Z \cap\left(S_{\perp} \cup E_{C}(X)\right)$; i.e. $F_{C}^{Z}\left(E_{C}^{*}(Z)\right)=E_{C}^{*}(Z)$ and for every $A \subseteq S$ such that $F_{C}^{Z}(A)=A$ we have $A \subseteq E_{C}^{*}(Z)$. Using $\nu$-notation, we can write:

$$
E_{C}^{*}(Z)=\nu X . F_{C}^{Z}(X)=\nu X . Z \cap\left(S_{\perp} \cup E_{C}(X)\right)
$$

Intuitively, $C$ is globally effective for $X$ at state $s$ if $s \in X$ and $C$ can

(C) Blackwell Publishers Lid and the Board of Trustees of the Bulletin of Economic Research 2001. 
subsequently maintain $X$; i.e. if $s$ is not a terminal state, $C$ is locally effective for $X$.

Often we are not interested in global effectivity generally but in terminal effectivity, the terminal states or final outcomes which a coalition can bring about. Analogous to program correctness two notions of effectivity can be distinguished. A coalition $C$ is partially effective for $X$ if it can guarantee $X$ to hold at every terminating (i.e. finite) run of the game/process. Thus, the strategy for $C$ may still lead to an infinite run. In contrast, $C$ is totally effective for $X$ if it is partially effective for $X$ and can guarantee termination at the same time. Formally, define $E^{p}, E^{\prime}: S \rightarrow(\mathscr{P}(N) \rightarrow \mathscr{P}(\mathscr{P}(S)))$ as the partial and total effectivity structures respectively as follows (where $\mu X$ denotes the least fixpoint):

$$
\begin{aligned}
& E_{C}^{p}(X)=E_{C}^{*}\left(\overline{S_{\perp}} \cup X\right)=\nu Y .\left(\overline{S_{\perp}} \cup X\right) \cap\left(S_{\perp} \cup E_{C}\left(Y^{\prime}\right)\right) \\
& E_{C}^{\prime}(X)=\mu Y .\left(S_{\perp} \cap X\right) \cup E_{C}(Y) .
\end{aligned}
$$

For total effectivity, we start with the terminal states which are in $X$ and iteratively add to them the states where $C$ can guarantee an outcome among those states. For partial effectivity, we require that $C$ is globally effective for $\overline{S_{\perp}} \cup X$; i.e. $C$ can maintain forcing states which are either nonterminal or in $X$.

While effectivity frames do not formally distinguish a special initial state $s_{0}$, in the modelling applications we shall often assume such a distinguished initial state; consequently, for example, the total effectivity function associated to such a frame will denote the total effectivity function of the initial state of the frame.

The following results capture the main relationships between global, partial and total effectivity. These results should be seen as generalizations of Dijkstra's (1976) work which discusses the links between partial and total program correctness.

Proposition 1:

1. For every effectivity frame gr $=(S, E)$, total effectivity implies partial effectivity; i.e. $E_{C}^{t}(X) \subseteq E_{C}^{p}(X)$.

2. For every superadditive effectivity firame $\mathscr{T}_{\mathrm{F}}=(S, E)$, at states where no infinile play is possible, partial effectivity intplies total effectivity; i.e. $E_{\not \varnothing}^{\prime}(S) \cap E_{C}^{\prime \prime}(X) \subseteq E_{C}^{\prime}(X)$.

3. For every C-regular and C-maximal effectivity frane gr $=(S, E)$, total effectivity is the clual of partial effectivity; i.e. $\overline{E_{C}^{\prime}(X)}=E_{\bar{C}}^{\prime \prime}(\bar{X})=$ $E_{\bar{C}}\left(\overline{\left.S_{\perp} \cap X\right)}\right.$.

Proof: (1) Let $\mathscr{F}_{r}=(S, E)$ be an effectivity frame. For any monotonic operator $F: \mathscr{P}(S) \rightarrow \mathscr{S}(S)$, define the upward and downward hierarchies 
as follows:

$$
\begin{aligned}
F \uparrow 0=\varnothing & F \downarrow 0=S \\
F \uparrow \kappa+1=F(F \uparrow \kappa) & F \downarrow \kappa+1=F(F \downarrow \kappa) \\
F \uparrow \lambda=\bigcup_{\kappa<\lambda} F \uparrow \kappa & F \downarrow \lambda=\bigcap_{\kappa<\lambda} F \downarrow \kappa
\end{aligned}
$$

where $\kappa$ and $\lambda$ are ordinals and $\lambda$ is a limit ordinal.

Given $X \subseteq S$, let $F_{t}(Y)=\left(S_{\perp} \cap X\right) \cup E_{C}(Y)$ and $F_{p}(Y)=\left(\overline{S_{\perp}} \cup X\right) \cap$ $\left(S_{\perp} \cup E_{C}(Y)\right)$. We show by transfinite induction that for all ordinals $\kappa$, $F_{l} \uparrow \kappa \subseteq F_{p} \downarrow \kappa$. For the inductive step:

$$
\begin{aligned}
F_{l} \uparrow \kappa+1 & =\left(S_{\perp} \cap X\right) \cup E_{C}\left(F_{l} \uparrow \kappa\right) \subseteq\left(\overline{S_{\perp}} \cup X\right) \cap\left(S_{\perp} \cup E_{C}\left(F_{p} \downarrow \kappa\right)\right) \\
& =F_{p} \downarrow \kappa+1
\end{aligned}
$$

using the induction hypothesis and monotonicity of $E_{C}$. For limit ordinals, the inductive step follows from the fact that for all $\kappa_{0} \leqslant \kappa$, $F \uparrow \kappa_{0} \subseteq F \uparrow \kappa$ and $F \downarrow \kappa \subseteq F \downarrow \kappa_{0}$. Now to prove the first claim, if $s \in E_{C}^{t}(X)$, there is some closure ordinal $\alpha$ such that for all $\beta \geqslant \alpha$, $s \in F_{l} \uparrow \beta$ and hence $s \in F_{p} \downarrow \beta$. Thus, for all ordinals $\gamma, s \in F_{p} \downarrow \gamma$, in particular for the closure ordinal $\gamma_{0}$ for which $F_{p} \downarrow \gamma_{0}=F_{p}\left(F_{p} \downarrow \gamma_{0}\right)=$ $E_{C}^{n}(X)$.

(2) Let $\mathscr{F}=(S, E)$ be a superadditive effectivity frame, and let $F_{f}(Y)=$ $S_{\perp} \cup E_{\varnothing}(Y)$. We show by induction that for all ordinals $\kappa, F_{f} \uparrow \kappa \cap$ $F_{p} \downarrow \kappa \subseteq F_{l} \uparrow \kappa$. For the inductive step $\kappa+1$, we must show that:

$$
\begin{aligned}
\left(S_{\perp} \cup E_{\varnothing}\left(F_{f} \uparrow \kappa\right)\right) \cap\left(\overline{S_{\perp}} \cup X\right) \cap\left(S_{\perp} \cup E_{C}\left(F_{\rho} \downarrow \kappa\right)\right) & \\
& \subseteq\left(S_{\perp} \cap X\right) \cup E_{C}\left(F_{\iota} \uparrow \kappa\right)
\end{aligned}
$$

In the case that $s \in E_{\varnothing}\left(F_{f} \uparrow r\right), s$ cannot be a terminal state and hence $s \in E_{C}\left(F_{p} \downarrow \kappa\right)$. Hence, by superadditivity, $s \in E_{C}\left(F_{f} \uparrow \kappa \cap F_{p} \downarrow \kappa\right)$ which by induction hypothesis and monotonicity implies that $s \in E_{C}\left(F_{l} \uparrow \kappa\right)$. The rest of the proof is analogous to the proof of (1).

(3)

$$
\begin{aligned}
E_{\bar{C}}^{*}\left(\overline{\left.S_{\perp} \cap X\right)}\right. & =\nu Y . \overline{S_{\perp} \cap X} \cap\left(S_{\perp} \cup E_{\bar{C}}(Y)\right) \\
& =\neg \mu Y .\left(S_{\perp} \cap X\right) \cup\left(\overline{S_{\perp}} \cap \overline{E_{\bar{C}}(\bar{Y})}\right) \\
& =\neg \mu Y .\left(S_{\perp} \cap X\right) \cup\left(\overline{\left.S_{\perp} \cap E_{C}(Y)\right)}\right.
\end{aligned}
$$

by $C$-maximality and regularity. Since $E_{C}(Y) \subseteq \overline{S_{\perp}}$, the last formula indeed equals $\overline{E_{C}^{\prime}(X)}$. 


\section{SPECIAL EFFECTIVITY FRAMES: GAMES}

Effectivity frames generalize many dynamic game models used in game theory. Below I consider in particular two classes of games, namely extensive games with and without simultaneous moves (the latter are simply extensive games of perfect information). As a particular example of simultaneous move games, I consider also democratic voting procedures. Note that in the games discussed in this paper, players have perfect information modulo simultaneous moves; i.e. the only source of imperfect information are simultaneous moves.

\section{III.1. Extensive games with simultaneous moves}

In an extensive game form with simultaneous moves (Osborne and Rubinstein, 1994), players may act simultaneously at every stage of the game in order to determine the resulting game position. Formally, to obtain such a model, we associate a strategic game with every state of the world. A strategic game $G=\left(N,\left\{\sum_{i} \mid i \in N\right\}, O, S\right)$ consists of the set of agents $N$, a nonempty set of strategies or actions $\sum S_{i}$ for every player $i \in N$, the set of states $S$ and an oulcome function $0: \prod_{i \in N} \sum_{i} \rightarrow S$ which associates with every tuple of strategies of the players (strategy profile) an outcome state in $S$.

In game theory (Binmore, 1992; Osborne and Rubinstein, 1994), strategic games also come equipped with a preference relation $\geqslant_{l} \subseteq S \times S$ for every player $i \in N$ which indicates which outcomes a player prefers. Strictly speaking, our strategic gannes are only game forms which can be turned into a game by adding these preference relations.

For notational convenience, let $\sigma_{C}:=\left\langle\sigma_{i}\right\rangle_{i \in C}$ denote the strategy tuple for coalition $C \subseteq N$ which consists of player $i$ choosing strategy $\sigma_{l} \in \sum_{i}$. Then given two strategy tuples $\sigma_{C}$ and $\sigma_{\bar{C}}$ (where $\left.\bar{C}:=N \backslash C\right), o\left(\sigma_{C}, \sigma_{\bar{C}}\right.$ ) denotes the outcome state associated with the strategy profile induced by $\sigma_{C}$ and $\sigma_{\bar{C}}$.

Let $\Gamma_{S}^{N}$ be the set of all strategic games between the set of players $N$ over the set of states $S$. Then we deline an extensive game (with simultaneous moves) as a partial function $\gamma: S \hookrightarrow \Gamma_{S}^{N}$ which associates strategic games to nonterminal states. An example of such a game will be looked at in Section IV.1.

The notion of effectivity which we associate with strategic games is known as $\alpha$-effectivity, (Moulin and Peleg, 1982; Moulin, 1983; Abdou and Keiding, 1991). Given a game $G$, a coalition $C \subseteq N$ will be $\alpha$-effective for a set $X \subseteq S$ iff the coalition has a joint strategy which will result in an outcome in $X$ no matter what strategies the other players choose. Formally, the $\alpha$ effectivity function $E_{G}^{\alpha}: \mathscr{P}(N) \rightarrow \mathscr{P}(\mathscr{P}(S))$ of a strategic game $G$ is defined as:

$$
X \in E_{G}^{\alpha}(C) \text { iff } \exists \sigma_{c} \forall \sigma_{\bar{C}} o\left(\sigma_{C,}, \sigma_{\bar{C}}\right) \in X
$$

(0) Blackwell Publishers L_td and the Board of Trustees of the Bulletin of Economic Rescarch 2001. 
We say that an effectivity function $E: \mathscr{P}(N) \rightarrow \mathscr{P}(\mathscr{P}(S)) \alpha$-corresponds to a strategic game $G$ if $E=E_{G}^{\alpha}$. Similarly, an effectivity frame $F=(S, E)$ $\alpha$-corresponds to an extensive game $\gamma: S \hookrightarrow \Gamma_{S}^{N}$ provided that $s E_{C} X$ iff $\gamma(s)$ is defined and $X \in E_{\gamma(s)}^{\mathrm{a}}$.

The question to be examined now is which effectivity frames $\alpha$ correspond to some extensive game. Call an effectivity function $E: P(N) \rightarrow \mathscr{P}(\mathscr{P}(S))$ playable iff it satisfies the following four conditions: (1) $\forall C \subseteq N: \varnothing \notin E(C),(2) \forall C \subseteq N: S \in E(C)$, (3) $E$ is $N$-maximal, and (4) $E$ is superadditive. Proposition 2 below extends characterization results obtained in by Moulin (1983) and Peleg (1998). A proof can be found in Pauly (2000).

Proposition 2: An effectivity function $E: \Phi(N) \rightarrow \Phi(\Phi(S)) \alpha$-corresponds to a strategic game $G \in \Gamma_{S}^{N}$ iff $E$ is playable.

Corollary 1: An effectivity frame $g_{H}=(S, E)$ cr-corresponds to an extensive game $\gamma: S \hookrightarrow \Gamma_{S}^{N}$ iff for every state $s \notin S_{1}, E(s)$ is playable.

I shall sometimes refer to an effectivity frame 9 simply as an extensive game if it $\alpha$-corresponds to such a game.

Claims (1) and (2) of Proposition 1 apply to extensive games, whereas claim (3) does not, since extensive games are not necessarily maximal. Consider, for example, the extensive game with simultaneous moves $\left(\left\{s_{0}, s_{1}, s_{2}, s_{3}, s_{4}\right\}, \gamma\right)$ where $\gamma$ is defined only for $s_{0}$ yielding the strategic game depicted in Figure 1.

Consider the effectivity frame $\mathscr{F}=(S, E)$ which $\alpha$-corresponds to this extensive game. Note that $E\left(s_{0}\right)$ is not maximal since the row player is not effective for $\left\{s_{1}, s_{4}\right\}$ while the column player is not effective for $\left\{s_{0}, s_{2}, s_{3}\right\}$ either. Furthermore, while the row player is not totally effective for $\left\{s_{1}, s_{4}\right\}$ at $s_{0}$, the column player is not partially effective for $\left\{s_{0}, s_{2}, s_{3}\right\}$ either.

\section{III.2. Extensive games without simultaneous moves}

A special case of the extensive games discussed in the previous section arises if at every stage of the game, one of the players is in complete control in determining the next stage. In such an extensive game without simultaneous moves, every stage of the game has a local dictator.

\begin{tabular}{|c|c|c|}
\hline \multirow{3}{*}{$\begin{array}{l}\text { up } \\
\text { down }\end{array}$} & left & right. \\
\hline & $s_{1}$ & $s_{2}$ \\
\hline & $s_{3}$ & $s_{4}$ \\
\hline
\end{tabular}

Fig. 1. A nommaximal game where partial and total effectivity are not duals (C) Blackwell Publishers Lld and the Board of Trustees of the Bulletin of Economic Rescarch 2001. 
Formally, we call a strategic game $G=\left(N,\left\{\sum_{i} \mid i \in N\right\}, O, S\right)$ a dictatorship iff there is some $d \in N$ such that:

$$
\forall S E \operatorname{ran}(o) \exists_{\sigma d} \forall \sigma_{N \backslash\{d\}} O(\sigma)=s
$$

In such a dictatorship, there is an individual $d$ (the dictator) whose choices completely determine the outcome state, independent of what the others do. Note that in case there is more than one dictator, the outcome function is constant (i.e. $\exists s \forall \sigma o(\sigma)=s$ ) and hence every player is a dictator.

Let $\Delta_{S}^{N}$ be the set of all dictatorships for the set of players $N$ over the set of states $S$. We define an extensive game without simultaneous moves as a partial function $\gamma: S \hookrightarrow \Delta_{S}^{N}$ which associates dictatorships to nonterminal states. Note that given an initial state, we can picture an extensive game without simultaneous moves as a standard game tree where nodes correspond to states which are labelled with the local dictator of that state; i.e. the player who is to move at that state. Figure 2 depicts an example.

At state $s_{0}$, player 1 is the local dictator who has the local power to decide between states $s_{1}$ and $s_{2}$. Similarly at state $s_{2}$, player 2 has the power to decide between states $s_{3}$ and $s_{4}$. Thus, states $s_{0}$ and $s_{2}$ are the nonterminal states to which $\gamma$ associates the two dictatorships given in the middle of Figure 2. The extensive game tree on the left can thus be viewed as a concise presentation of a sequence of local dictatorships. The example also shows that a sequence of local dictatorships need not result in a dictatorship overall, as the strategic form of the extensive game illustrates where neither player is a dictator. Note that in case $|N|=1$ or in case the local dictator is the same at every state, the game is a oneplayer game, or equivalently, a process.

Call an effectivity function $E: \mathscr{P}(N) \rightarrow \mathscr{P}(\mathscr{P}(S))$ individualistic iff it is playable and $E(N)=\cup_{i \in N} E(\{i\})$. The condition ensures that everything which can be forced can be forced already by some individual. The following result (proved in Pauly, 2000) shows that individualism is an extremely strong assumption: While it seems to say only that the whole is equal to the sum of its parts, due to superadditivity, it actually says that the whole is equal to one particular part.

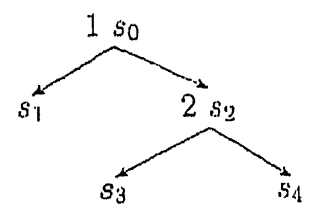
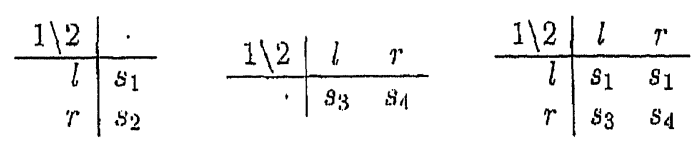

Fig. 2. An cxtensive game (left) without simultaneous moves which can be viewed as the sequential composition of two local dictatorships (middle); the strategic form of this extensive game (right) on the other hand is not a diclatorship 
Proposition 3: An effectivity function $E: \mathscr{P}(N) \rightarrow \mathscr{\Im}(\mathscr{P}(S))$ a-corresponds to a dictatorship $G \in \Delta_{S}^{N}$ iff $E$ is individualistic.

Put positively, unless we have a dictatorship, coalitions of agents can sometimes achieve more than their members individually; cooperation is thus advantageous.

Corollary 2: An effectivity fiame Gr $=(S, E) \alpha$-corresponds to an extensive game without simultaneous moves $\gamma: S \hookrightarrow \Delta_{S}^{N}$ iff for every state $s \notin S_{\perp}$, $E(s)$ is individualistic.

Since individualistic effectivity functions are regular and maximal, all three claims of Proposition 1 apply to extensive games without simultaneous moves.

\section{III.3. Democracies}

Effectivity frames can also be utilized to model the power of coalitions in voting procedures. Consider a strategic game $G=\left(N,\left\{\sum_{i} \mid i \in N\right\}, O, S\right)$ where $|N|$ is odd, $\sum_{i}=\{y e s, n o\}$ for all $i \in N$, and there are states $s_{y}, s_{n} \in S$ (we allow for $s_{j}=s_{n}$ ) such that for every strategy profile $\sigma$, $o(\sigma) \in\left\{s_{y}, s_{n}\right\}$ and $o(\sigma)=s_{y}$ if $\left|\left\{\sigma_{i} \mid \sigma_{i}=y e s\right\}\right|>\frac{1}{2}|N|$ and $o(\sigma)=s_{n}$ otherwise. This strategic gane corresponds to a vote between two alternatives where each member of $N$ participates and the outcome state is determined by the majority of the votes. We shall call such a voting game a two-alternative majority vote. Since an even number of voters creates the problem of how to resolve ties, we require $|N|$ to be odd here. Nonetheless, this simple example should demonstrate how results for more elaborate 'democratic' voting procedures (e.g. including a distinguished chairman who decides in case of a tie) can be obtained.

Call an effectivity function $E: \mathscr{P}(N) \rightarrow \mathscr{P}(\mathscr{P}(S)$ ) majorative iff for every coalition $C$ with $|C|>\frac{1}{2}|N|$ we have $E(N) \subseteq E(C)$. $E$ is binary iff:

$$
X \in E(N) \quad \text { and } \quad \bar{X} \cap Y \in E(N) \Rightarrow X \cup Y \in E(\varnothing)
$$

Quite naturally, the first condition formalizes that a majority suffices to establish anything, and the second condition captures that there are only two alternatives to be chosen from. Note that if $E$ is playable and majorative, $E$ is also maximal and furthermore $\bar{X} \in E(N) \Rightarrow X \notin E(C)$ for $|C|<\frac{1}{2}|N|$.

Proposition 4: An effectivity function $E: \mathscr{P}(N) \rightarrow \mathscr{P}(\mathscr{P}(S)) \alpha$-corresponds to a two-alternative majority vote $G=\left(N,\left\{\sum_{i} \mid i \in N\right\}, O, S\right)$ iff $E$ is playable, majorative and binary.

(C) Blackwell Publishers Ltd and the Board of Trustecs of the Bulletin of Economic Research 2001. 
Proof: It is easily verified that the $\alpha$-effectivity function of any twoalternative majority vote is majorative and binary. For the other direction, let $E$ be any playable, majorative and binary effectivity function. By playability, $E=E_{G}^{\alpha}$ for some strategic game $G$. We shall construct a two-alternative majority vote $G^{\prime}$ such that $E=E_{G}^{\alpha}=E_{G^{\prime}}^{\alpha}$.

In case the outcome state in $G$ is always the same state $s$, constructing $G^{\prime}$ is trivial. So assume there are at least two outcomes $s_{1}, s_{2} \in S$ which actually occur in $G$. Then simply define $G^{\prime}=\left(N,\left\{\sum_{i} \mid i \in N\right\}, O, S\right)$ with $\sum_{i}=\{$ yes, no $\}$ and $o(\sigma)=s_{1}$ if $\left|\left\{\sigma_{i} \mid \sigma_{l}=y e s\right\}\right|>\frac{1}{2}|N|$ and $o(\sigma)=s_{2}$ otherwise. Observe first that since $E$ is binary, $G$ cannot result in an outcome other than $s_{1}$ or $s_{2}$ : from $\left\{s_{1}\right\} \in E(N)$ and $\left\{s_{2}\right\} \in E(N)$ we can conclude that $\left\{s_{1}, s_{2}\right\} \in E(\varnothing)$. It remains to show that $E_{G}^{\alpha}=E_{G^{\prime}}^{\alpha}$.

If $X \in E_{G}^{\alpha}(C)=E(C)$, there are three possibilities: (i) $\left\{s_{1}, s_{2}\right\} \subseteq X$, in which case $X \in E_{G^{\prime}}^{\alpha}(C)$ holds trivially. (ii) $s_{2} \notin X$, and so $s_{1} \in X$. Then $|C|>\frac{1}{2}|N|$, for otherwise since $E$ is majorative, $\bar{X} \notin E(N)$, contradicting the fact that $\left\{s_{2}\right\} \in E(N)$. So since $C$ forms a majority, $X \in E_{G^{\prime}}^{\prime(}(C)$. (iii) $s_{1} \notin X$, analogously.

For the other direction, if $X \in E_{G^{\prime}}^{\alpha}(C)$, we can distinguish the same three cases: (i) $\left\{s_{1}, s_{2}\right\} \subseteq X$, in which case monotonicity and $\left\{s_{1}, s_{2}\right\} \in E(\varnothing)$ establish that $X \in E(C)$. (ii) $s_{2} \notin X$, and so $s_{1} \in X$. Then $C$ must be a majority, and so by majorativity, $X \in E(C)$. (iii) $s_{1} \notin X$, analogously.

Defining a democratic binary procedure as a partial function which associates two-alternative majority votes to nonterminal states, we obtain the following:

Corollary 3: An effectivity frame $F=(S, E) \alpha$-corresponds to a democratic binary procedure iff for every state $s \notin S_{\perp}, E(s)$ is playable, majorative and binary.

Since playable majorative effectivity functions are also maximal, Proposition 1 applies to democracies as well.

\section{IV, SOME APPLICATIONS}

\section{IV.1. Modelling dynamic procedtures: voling}

Consider a political body $N=\{1,2,3,4,5,6\}$ which has to decide on passing a new law. First, a subcommittee $D=\{2,3,4\}$ has to decide (by majority) which precise version of the law is to be presented to the full political body. Subsequently, the whole political body decides whether the law is passed or not. Again, the majority of the votes decides, and in case of a draw, the vote of the chaiman 1 is decisive. If the law (as 
proposed by committee $D$ ) is not passed, the initiative is returned to committee $D$ which has to make a new proposal for the law, and the process repeats itself.

We assume for simplicity that there are only two versions of the law which are under discussion, version 1 and version 2 . If the body $N$ rejects the proposal of committee $D$, the committee can either decide to propose the other version of the law, or it can resubmit its original proposal, possibly resulting in a stalemate which may turn into an infinite loop (some might claim that this model is sufficiently realistic to capture the essentials of the legislative process in some countries). Figure 3 depicts the situation as a tree.

The situation described is easily seen to be an extensive game with simultaneous moves. One can think of the situation described in terms of coalitional effectivity as follows: $s_{0} E_{C} X$ holds iff at state $s_{0}$, coalition $C$ can force the local voting outcome to lie in set $X$, i.e. iff one of the following two conditions is met: (1) $\{t, u\} \cap X \neq \varnothing$ and $|C \cap D|>1$, or (2) $\{t, u\} \subseteq X$. Analogous definitions can be given for $t E_{C} X$ and $u E_{C} X$, incorporating the special role of the chairman.

We can also use this example to illustrate global, partial and total effectivity. Figure 4 displays some interesting examples which demonstrate the unequal powers of four 3-player coalitions at the initial state $s$.

Note that both $\{1,2,3\}$ and $\{1,4,5\}$ can globally maintain $\left\{s_{0}, t, u\right\}$; i.e. they have the power to keep the process going forever, never reaching any decision. On the other hand, $\{1,4,5\}$ cannot force a particular version of the law to be passed, whereas $\{2,3,4\}$ does have at least partial power to do so: If some mechanism in the legislative process would rule out an infinite stalemate, this coalition can guarantee any outcome; still, it is unable to rule out such an infinite stalemate on its

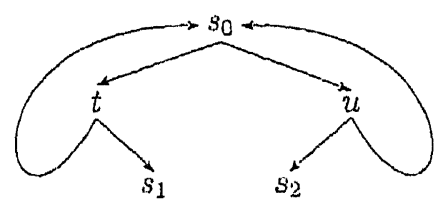

by majority of $D$

by majority of $N$

Fig. 3. An example of binary majority voting with subcommittees

\begin{tabular}{r|ccc} 
coalition/states & $\left\{s_{1}\right\}$ & $\left\{s_{1}, s_{2}\right\}$ & $\left\{s_{0}, t, u\right\}$ \\
\hline$\{1,2,3\}$ & $t$ & $t$ & $g$ \\
$\{2,3,4\}$ & $p$ & $p$ & - \\
$\{1,4,5\}$ & - & $t$ & $g$ \\
$\{4,5,6\}$ & - & - & -
\end{tabular}

Fig. 4. Global (g), total $(\mathrm{t})$ and partial $(\mathrm{p})$ effectivity in the voting example of Fig. 3 state so

(0) Blackwell Publishers Led and the Board of Trustces of the Bulletin of Econonic Rescarch 2001. 
own; i.e. it is partially but not totally effective for $\left\{s_{1}\right\}$ as well as for $\left\{s_{2}\right\}$. Thus, the following partial effectivity order of these four three-player coalitions emerges:

$$
\{4,5,6\}<\{2,3,4\},\{1,4,5\}<\{1,2,3\} .
$$

\section{IV.2. Local vs. global properties}

The different kinds of effectivity frames associated, for example, with extensive games and democracies have been defined in terms of local requirements; i.e. properties which the local effectivity functions had to satisfy. Some of these properties will be maintained globally or terminally, some will not. One can show, for example, that for games without infinite plays, the total (= partial) effectivity function is playable.

Proposition 5: If $\mathscr{T}_{P}=(S, E)$, o-corresponds to an extensive game and $s \in E_{\varnothing}^{\prime}(S)$, then $E^{t}(s)$ is playalule.

Proof: The playability conditions can be checked one-by-one; we only show $N$-maximality and superadditivity. If $s \notin E_{\varnothing}^{l}(\bar{X})$, since $s \in E_{\varnothing}^{\prime}(S)$, we have $s \notin E_{\varnothing}^{p}(\bar{X})$ by Proposition 1; i.e. $s \in E_{N}^{*}\left(S_{\perp} \cap X\right)=E_{N}^{l}(X)$.

For superadditivity, we show generally that for all $C_{1} \cap C_{2}=\varnothing$ we have $E_{C_{1}}^{t}\left(X_{1}\right) \cap E_{C_{2}}^{\prime}\left(X_{2}\right) \subseteq E_{C_{1}}^{l} \cup C_{2}\left(X_{1} \cap X_{2}\right)$; we proceed again by transfinite induction as in the proof of Proposition. Let:

$$
\begin{aligned}
& F_{1}(Y)=\left(S_{\perp} \cap X_{1}\right) \cup E_{C_{1}}(Y) \\
& F_{2}(Y)=\left(S_{\perp} \cap X_{2}\right) \cup E_{C_{2}}(Y) \\
& F_{3}(Y)=\left(S_{\perp} \cap X_{1} \cap X_{2}\right) \cup E_{C_{1} \cup C_{2}}(Y) .
\end{aligned}
$$

We show that $F_{1} \uparrow \kappa \cap F_{2} \uparrow \kappa \subseteq F_{3} \uparrow \kappa$. The heart of the proof is the inductive step for $k+1$, where one can check that:

$$
\left(\left(S_{\perp} \cap X_{1}\right) \cup E_{C_{1}}\left(F_{1} \uparrow \kappa\right)\right) \cap\left(\left(S_{\perp} \cap X_{2}\right) \cup E_{C_{2}}\left(F_{2} \uparrow \kappa\right)\right)
$$

is a subset of

$$
\left(S_{\perp} \cap X_{1} \cap X_{2}\right) \cup E_{C_{1} \cup C_{2}}\left(F_{3} \uparrow \kappa\right) .
$$

The only two cases possible are $s \in\left(S_{\perp} \cap X_{1}\right) \cap\left(S_{\perp} \cap X_{2}\right)$ and $s \in E_{C_{1}}\left(F_{1} \uparrow \kappa_{i}\right) \cap E_{C_{2}}\left(F_{2} \uparrow \kappa\right)$. The latter case makes use of the superadditivity of $E$.

Consequenuly, for every extensive game $G$ without infinite plays, there is a strategic game $G^{\prime}$ such that the total effectivity function of $G$ is the $\alpha$ effectivity function of $G^{\prime}$. In fact, one such strategic game $G^{\prime}$ is simply 
the strategic normal form of $G$ (see Osborne and Rubinstein, 1994). Note also that the global effectivity function $E^{*}$ is not playable: In the simple one-player game where at the initial state $s$, the player has only one possible move resulting in a final state $t, E^{*}$ is not $N$-maximal: neither $s E$ 光 $\left(\overline{S_{\perp}}\right)$ nor $s E_{N}^{*}\left(S_{\perp}\right)$ holds.

Considering democracy as a further example, while the binary aspect of a democratic binary procedure may be lost globally, the democratic aspect is maintained: If $E$ is majorative at every state of an effectivity frame, then so is $E^{\prime}$, so a democratic procedure will maintain democracy overall. That the converse is not true can be gathered from the extensive game in Figure 5 where at the initial state, $E^{t}$ is majorative while $E$ is not.

\section{IV.3. Realization problems}

Given a particular effectivity function, one may ask whether it can be implemented or realized by means of a particular procedure. An agenda setter might be interested to find out whether a given power distribution can be realized by a (democratic) voting procedure of a particular kind. More abstractly, given a strategic game $G$ with its associated $\alpha$-effectivity function $E_{G}^{u}$, one might want to know whether there is an extensive game without simultancous moves $G^{\prime}$ such that $E_{G^{\prime}}^{\prime}=E_{G}^{\mathrm{ck}}$.

As a very simple example of a realization problem using $\alpha$-effectivity, consider again the strategic game form $G$ of Figure 1. Since there is no extensive game $G^{\prime}$ without simultaneous moves such that $E_{G^{\prime}}^{t}=E_{G^{\alpha}}^{\alpha}$, there can also be no extensive game with a reduced strategic form equivalent to $G$. In contrast, if we replace $s_{4}$ by $s_{3}$, the extensive game $G^{\prime}$ of Figure 6 satisfies $E_{G^{\prime}}^{l}=E_{G}^{\alpha}$ (player 1 plays rows, player 2 columns) and has an equivalent reduced strategic form.

In general, the target effectivity function does not need to be specified completely, one may only be interested that it meets certain criteria such as being majorative. This suggests that the realization problem can be considered as a generalization of the program synthesis problem; i.e. of finding a program which meets a given specification. Thus, in the oneplayer case, the realizing effectivity frame is a process satisfying/implementing the specification.

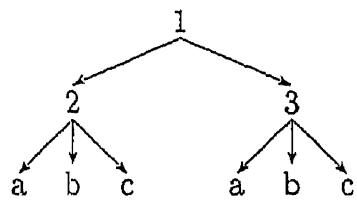

Fig. 5. A game which is majorative terminally but not locally

(c. Blackwell Publishers Lid and the Board of Trustees of the Bulletin of Economic Rescarch 2001. 
<smiles>CCCC[13CH]</smiles>

Fig. 6. An extensive game without simultaneous moves<smiles>CCC(C)C</smiles>

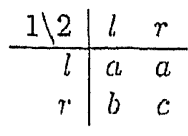<smiles>CCCCCC(C)C</smiles>

\begin{tabular}{r|rr}
$1 \backslash 2$ & $l$ & $r$ \\
\hline$l l$ & $a$ & $a$ \\
$l r$ & $a$ & $c$ \\
$r l$ & $b$ & $a$ \\
$r r$ & $b$ & $c$
\end{tabular}

Fig. 7. Two extensive games

The question, whether the $c x$-effectivity function of a strategic game can be realized by an extensive game, is closely related to a question that has received some attention in the game theory literature (see e.g., Abdou, 1998). For which strategic game forms does there exist an extensive game form (of perfect information, without simultaneous moves) with the same reduced strategic form? Two strategic games with the same reduced strategic form have the same $\alpha$-effectivity function while the converse does not hold, as the two extensive games in Figure 7 show.

The table to the right of each game represents its (reduced) strategic form. From the perspective of $\alpha$-effectivity, both extensive games are the same, whereas the strategic form of the game on the right reveals that player 1 has strategic options unavailable to him in the game on the left. In the right game, player 1 has a strategy $l r$ which guarantees him either outcome $a$ or outcome c. From the point of view of $\alpha$-effectivity, this strategy is negligible given that he has a 'stronger' strategy $I l$ which guarantees outcome $a$. Still, player 1 maly prefer strategy $l r$ which forces $\{a, c\}$ to strategy $l l$ which forces $\{a\}$ if he strictly prefers $c$ to $a$. So from a perspective which includes preferences, one maly not want to identify the two games.

\section{A LOGICAL FRAMEWORK}

\section{V.1. Syntax and semantics}

Coalition logic, introduced in Putuly (2000), provides a formal system to reason about effectivity frames. The formula $[C] \varphi$ is true at a state provided that coalition $C$ is locally effective for achieving a state where $\varphi$ holds. In this paper, I extend the purely modal system by adding a new operator for global effectivity: [ $\left.C^{*}\right] \varphi$ is true at a state if coalition $C$ is 
globally effective for $\varphi$. The resulting logic can be viewed as a generalized multiplayer version of the game logic proposed by Parikh (1985).

Given the set of agents $N$, we define the syntax of coalition logic as follows. Given a set of atomic propositions $\Phi_{0}$, a formula $\varphi$ can have the following syntactic form:

$$
\varphi:=\perp|p| \neg \varphi|\varphi \vee \varphi|[C] \varphi \mid\left[C^{*}\right] \varphi
$$

where $p \in \Phi_{0}$ and $C \subseteq N$. We define $T, \wedge, \rightarrow$ and $\leftrightarrow$ as usual: $T:=\neg \perp$, $\varphi \wedge \psi:=\neg(\neg \varphi \vee \neg \psi), \varphi \rightarrow \psi:=\neg \varphi \vee \psi$ and $\varphi \leftrightarrow \psi:=(\varphi \rightarrow \psi) \wedge(\psi \rightarrow \varphi)$. In case $C=\{i\}$, we write $[i] \varphi$ instead of $[\{i\}] \varphi$. Furthermore, we define terminal effectivity in terms of global effectivity as is to be expected given the semantic definitions of $E^{t}$ and $E^{p}$, i.e. we use the following abbreviations:

$$
\begin{aligned}
\sigma_{\perp} & :=\wedge C \subseteq N \neg[C] \top \\
{\left[C^{p}\right] \varphi } & :=\left[C^{*}\right]\left(\varphi \vee \neg \sigma_{\perp}\right) \\
{\left[C^{\prime}\right] \varphi } & :=\neg\left[\bar{C}^{*}\right] \neg\left(\varphi \wedge \sigma_{\perp}\right)
\end{aligned}
$$

Note that the definition of $\left[C^{l}\right] \varphi$ relies on Proposition 1 which applies only to regular and maximal frames, but since all of the frames we will be dealing with in our applications fall into that category, we can cut back on the number of primitive operators.

An effectivity model $M=(S, E, V)$ consists of an effectivity frame $\mathscr{F}_{F}=(S, E)$ and a valuation for the propositional letters $V: \Phi_{0} \rightarrow \mathscr{P}(S)$. Given such a model, truth of a formula in a model at a state is defined as follows:

$$
\begin{aligned}
M, s \not 1 & \\
M, s \neq p & \text { iff } p \in \Phi_{0} \text { and } s \in V(p) \\
\mathcal{M}, s \vDash \neg \varphi & \text { iff } \mathcal{M}, s \neq \varphi \\
\mathcal{M}, s \vDash \varphi \vee \psi & \text { iff } M, s \vDash \varphi \text { or } M, s \vDash \psi \\
\mathcal{M}, s \vDash[C] \varphi & \text { iff } s E_{C} \varphi^{M} \\
\mathcal{M}, s \vDash\left[C^{*}\right] \varphi & \text { iff } s E_{C}^{*} \varphi^{M}
\end{aligned}
$$

where $\varphi^{\mathcal{M}}=\{s \in S \mid \mathcal{M}, s \neq \varphi\}$. A formula $\varphi$ is valid in $\mathcal{M}$ iff $\varphi^{\mathcal{M}}=S$, and $\varphi$ is valid iff $\varphi$ is valid in all effectivity models. A set of formulas $\Psi$ is satisfiable iff there is a model $\mathcal{M}$ for which $\cap_{\psi \in \Psi} \psi^{\mathcal{M}} \neq \varnothing$.

\section{V.2. Applications}

The purpose of this section is to show how the three applications illustrated in Section IV can all be translated into logical questions of model checking, validity and satisfiability.

(c): Blackwell Publishers Letd and the Boalrd of Trustecs of the Bulletin of Economic Research 2001. 
Reconsidering first the voting procedure of Section IV.1, one can easily translate the facts about global and terminal effectivity into our logical language. Let $M=(S, E, V)$ be the model which captures the procedure depicted in Figure 3, where $\Phi_{0}=\left\{p_{1}, p_{2}, q\right\}$ and $V\left(p_{i}\right)=\left\{s_{i}\right\}$, and $V(q)=\left\{s_{0}, l, u\right\}$. Then at the initial state, $M, s_{0} \vDash\left[\{1,2,3\}^{t}\right] p_{1} \wedge$ $\left[\{1,2,3\}^{t}\right] p_{2} \wedge\left[\{1,2,3\}^{*}\right] q$; i.e. the coalition $\{1,2,3\}$ can achieve any possible outcome as well as a stalemate, whereas the coalition $\{1,4,5\}$ is weaker, $\mathcal{M}, s_{0} \vDash\left[\{1,4,5\}^{\prime}\right]\left(p_{1} \vee p_{2}\right)$ but $\mathcal{M}, s_{0} \not \forall\left[\{1,4,5\}^{\prime}\right] p_{1} \vee$ $\left[\{1,4,5\}^{t}\right] p_{2}$. Furthermore, $\mathcal{M}, s_{0}=\left[\{1,4,5\}^{*}\right] q$, so this coalition can block any law from getting passed. Even weaker, coalition $\{4,5,6\}$ has virtually no power, since its counter-coalition $\{1,2,3\}$ is all-powerful. Thus, these facts about truth in al given model are the logical analogue of Figure 4.

As for the logical analogue of Proposition 5, the preservation of local properties on the global level, observe that the four playability conditions for $E^{\prime}$ can be translated into the logical language:

( $\perp$ ) $\neg\left[C^{l}\right] \perp$

(T) $[N] T \rightarrow\left[C^{\prime}\right] T$

(N) $[N] T \rightarrow\left(\neg\left[\varnothing^{t}\right] \neg \varphi \rightarrow\left[N^{t}\right] \varphi\right)$

(S) $\quad\left(\left[C_{1}^{t}\right] \varphi_{1} \wedge\left[C_{2}^{t}\right] \varphi_{2}\right) \rightarrow\left[\left(C_{1} \cup C_{2}\right)^{\prime}\right]\left(\varphi_{1} \wedge \varphi_{2}\right)$

where $C_{1} \cap C_{2}=\varnothing$.

Note that the antecedent of axioms $(T)$ and $(N)$ is necessary to handle terminal states where no coalition is effective for anything. All four axiom schemas are valid for extensive games without infinite plays. Similarly, the majorativity condition can be translated into:

$$
\left[N^{t}\right] \varphi \rightarrow\left[C^{t}\right] \varphi
$$

where $|C|>\frac{1}{2}|N|$, and can be shown to be valid for majorative effectivity frames.

Lastly, the realization problem of Section IV.3 can be formulated axiomatically as well. We examine again the strategic game $G$ of Figure 1 with $N=\{1,2\}$ and its associaled $\alpha$-effectivity function $E_{G}^{\alpha}$. We use $\Phi_{0}=\{a, b, c, d\}$ for the outcomes of the game and specify that the outcomes are mutually exclusive, complete and hold only at terminal states:

$$
\begin{gathered}
{\left[\varnothing^{*}\right](a \rightarrow \neg b \wedge \neg c \wedge \neg c)} \\
{\left[\varnothing^{*}\right](b \rightarrow \neg a \wedge \neg c \wedge \neg c)} \\
{\left[\varnothing^{*}\right](c \rightarrow \neg a \wedge \neg b \wedge \neg c)} \\
{\left[\varnothing^{*}\right](d l \rightarrow \neg a \wedge \neg b \wedge \neg c)} \\
{\left[\varnothing^{*}\right]\left(\sigma_{\perp} \leftrightarrow(a \vee b \vee c \vee d)\right) .}
\end{gathered}
$$

6. Blackwell Publishers Lid and the Board of Trustees of the Bulletin of Economic Research 2001. 
Next, we require that all outcomes are possible in the game:

$$
\left[N^{t}\right] a \wedge\left[N^{t}\right] b \wedge\left[N^{t}\right] c \wedge\left[N^{t}\right] d
$$

and that there are no infinite plays allowed:

$$
\left[\varnothing^{t}\right] T
$$

Finally, we specify the target effectivity function of the strategic game:

$$
\left[1^{t}\right](a \vee b) \wedge\left[1^{\prime}\right](c \vee d) \wedge\left[\underline{2}^{t}\right](a \vee c) \wedge\left[2^{t}\right](b \vee d)
$$

Let $\Delta$ be the set consisting of these eight axioms. Then $\Delta$ is satisfiable by an extensive game without simultaneous moves iff $E_{G}^{\alpha}$ is realized by this game. Hence, $\Delta$ is not satisfiable by an extensive game without simultaneous moves. On the other hand, considering $G^{\prime}$ with $s_{4}$ being replaced by $s_{3}$ in $G$ and substituting $c$ for $d$ in the axioms appropriately yielding the set $\Delta^{\prime}$, the extensive game of Figure 6 satisfies $\Delta^{\prime}$.

\section{METATHEORY}

The concern of the present paper has been mainly semantic in nature. I introduced a class of models and discussed various well-known subclasses, also showing how these models can be used to model scenarios of multiagent interaction and what new questions are raised. On the logical side, there are a number of metatheoretic questions which should be mentioned. In Pauly (2000), the modal base logic (i.e. coalition logic without $\left[C^{*}\right] \varphi$ ) has been studied from a more logical perspective. As has been suggested in the previous section, the playability conditions can be translated into our logical language yielding an axiomatization which can be shown to be complete for the class of extensive games with simultaneous moves. Work is in progress on extending this axiomatization to the logic with $\left[C^{*}\right] \varphi$. Furthermore, the satisfiability problem for the modal base logic was shown to be PSPACE-complete, and hence it is just as complex as the normal modal logic $K$. In contrast, introducing an iteration construct such as $\left[C^{*}\right] \varphi$ usually increases the complexity of the satisfiability problem. In the cise of propositional dynamic logic (Harel, 1984; Kozen and Tiuryn, 1990), the satisfiability problem is exponentialtime complete, and I conjecturc that the same is true for coalition logic.

\section{CONCLUSIONS AND FUTURE WORK}

For the computer scientist, the work presented in this paper should provide an interesting generalization of work initiated by Dijkstra (1976) on partial and total program correctness. Moving from programs to 
multiplayer processes, one gets a better picture of the assumptions needed to establish various connections between partial and total correctness (Proposition 1). For the game-theorist on the other hand, the distinction between partial and total effectivity provides the conceptual tool to analyse situations where certain coalitions have the power to force an impasse through infinite looping. Furthermore, I hope to have shown that effectivity frames are a useful model for dynamic processes and raise some new questions as well. The fact that a logic can be associated to these effectivity frames provides not only a conceptual link between game theory and logic, but also an algorithmic approach to solving game-theoretic questions.

Besides the open metatheoretic questions mentioned, there are also game-theoretic questions which lend themselves for future work. The literature on effectivity functions knows various concepts of effectivity which differ from the notion of $\alpha$-effectivity employed here. The general approach adopted here equally well applies to these other notions such as $\beta$-effectivity, since effectivity [rames, global and terminal effectivity are not formally tied to any particular notion of effectivity.

More fundamental still, the present logic does not allow one to talk about players' preferences. Put differently, we can only express what players and coalitions can achieve, not what they want to achieve. Moving from the possible to the desirable is needed in order to incorporate various solution concepts such as the core so that we can eventually add predicates such as $\operatorname{Core}(\varphi)$ expressing that $\varphi$ holds at all states/outcomes in the core. To add preferences, one can add a standard modal accessibility relation for each player together with a new modal operator $\operatorname{Pre}_{i}(\varphi)$ which says that at all states preferred by $i, \varphi$ holds. It remains to be seen whether such a modal operator is sufficient to formalize various solution concepts, for it may turn out that a richer fragment of first-order logic is needed to compare preferences globally.

On a more applied note, examples such as the legislative procedure given in Section IV.1 suggest looking at more realistic cases of political/social processes where coalition formation is involved. As shown by, for example, Vannucci (2000), effectivity functions can play a role in such an analysis, and it would be interesting to see how effectivity frames and coalition logic could be useful here.

\section{REFERENCES}

Abdou, J. (1998). 'Rectangularity and tightness: a normal form characterization of perfect information extensive game forms', Mathematics of Operations Research, vol. 23, no. 3, pp. 553-67.

Abdou, J, and Keiding, H. (1991). Effectivity Functions in Social Choice. Kluwer. Binmore, K. (1992). Fun and Games:' A Text on Game Theorys. Heath.

(C) Blackwell Publishers Lid and the Board of Trustees of the Bulletin of Economic Rescarch 2001. 
Dijkstra, E. (1976). A Discipline of Programming, Prentice-Hall.

Harel, D. (1984). 'Dynamic logic', in: Gabbay, D. and Guenthner, F. (eds), Handbook of Philosophical Logic, vol. II. Reidel.

Kozen, D. and Tiuryn, J. (1990). 'Logics of programs', in: Jan van Leeuwen (ed.), Handbook of Theoretical Computer Science, vol. B. MIT Press.

Moulin, H. (1983). The Strategy of Social Choice. North-Holland.

Moulin, H. and Peleg, B. (1982). 'Cores of effectivity functions and implementation theory', Journal of Mathematical Economics, vol. 10, no. 1, pp. 115-45.

Osborne, M. and Rubinstein, A. (1994). A Course in Game Theory, MIT Press.

Parikh, R. (1985). 'The logic of games and its applications', in: Karpinski, M. and van Leeuwen, J. (eds), Topics in the Theory of Compulation, vol. 24 of Annals of Discrete Mathematics. Elsevier.

Pauly, M. (2000). 'A modal logic for coalitional power in games', Journal of Logic and Computation (in press).

Peleg, B. (1998). 'Effectivity functions, game forms, games and rights', Social Choice and Welfare, vol. 15, pp. 67-80.

Vannucci, S. (2000). 'Effectivity functions and parliamentary governance structures', in: Patrone, F., Garcia-Jurado, I. and Tijs, S. (eds), Game Practice: Contributions: from Applied Game Theor's, pp. 225-39. Kluwer. 